\title{
Qualidade de vida, depressão e cognição a partir da educação gerontológica mediada por uma rádio-poste em instituições de longa permanência para idosos
}

\author{
Quality of life, depression and cognition based on gerontological education mediated by a pole \\ radio in homes for the aged
}

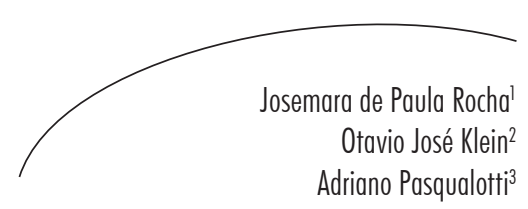

\section{Resumo}

Objetivo: identificar possíveis mudanças na qualidade de vida, cognição e depressão advindas de oficinas de educação gerontológica mediadas por uma rádio-poste em instituições de longa permanência para idosos. Métodos: estudo observacional, de natureza quali-quantitativa, que contemplou variáveis descritivas e de associação. Após estudo piloto em um grupo de terceira idade, foram selecionadas por conveniência quatro instituições, e delas os idosos cujas características contemplavam os critérios de inclusão. Foram aplicados questionários relacionados com qualidade de vida, depressão, estado cognitivo, características sociodemográficas e realidade tecnológica. Para analisar os efeitos pré e pós-processo de intervenção, foram realizadas estatísticas descritiva e analítica, por meio dos testes não paramétricos de Mann-Whitney, Kolmogorov-Smirnov, Exato de Fisher, Alfa de Cronbach, Correlação linear de Spearman, todos com nível de significância de $\mathrm{p} \leq 0,05$. Foram elaborados roteiros de rádio para as oficinas, que aconteceram em oito encontros em cada instituição, duas vezes semanais, duas horas cada, durante seis meses. Resultados: ocorreram incrementos na qualidade de vida e cognição, de forma estatisticamente significativa nos domínios "recreação", "intimidade" e na "memória imediata". Conclusões: os ganhos significativos em qualidade de vida e cognição sugerem que essa prática pode ser válida para melhorar as condições de saúde de idosos de instituições de longa permanência.

\section{Abstract}

Objective: to identify the changes in the quality of life, cognition and depression from gerontological education workshops mediated by a pole radio in long-term institutions for the aged. Methodology: Observational qualitative and quantitative study, including

\footnotetext{
Programa de Residência Multiprofissional Integrada em Saúde do Idoso e Atenção ao Câncer. Universidade de Passo Fundo. Passo Fundo, RS, Brasil.

Faculdade de Artes e Comunicação. Universidade de Passo Fundo. Passo Fundo, RS, Brasil.

Programa de Pós-graduação em Envelhecimento. Universidade de Passo Fundo. Passo Fundo, RS, Brasil.
}

Palavras-chave: Saúde do Idoso Institucionalizado. Meios de Comunicação. Relações Interpessoais Qualidade de vida. Cognição. Depressão.

Financiamento da pesquisa: o projeto de pesquisa recebeu apoio financeiro mediante edital Fapergs 003/2009 ARD processo 0902756 e MCT/CNPq processo 478530/2009-9. 
descriptive and association variables. After a pilot study with an elderly group we selected four institutions for convenience and the elderly whose characteristics met the inclusion criteria. We applied questionnaires related to quality of life, depression, cognitive status, socio-demographic characteristics and technological reality. To analyze the effects of pre-and post-intervention process, descriptive and analytical statistics were performed, making the nonparametric Mann-Whitney, KolmogorovSmirnov test, Fisher exact, Cronbach's alpha, linear Spearman correlation, all of them with level significance of $\mathrm{p} \leq 0.05$. We developed radio scripts for the workshops, held eight meetings in each institution, twice a week, two hours each, for six months. Results: There were increases in the life quality and cognition, in a statistically significant way in the domains classified as recreation, intimacy, and in the immediate memory. Conclusion: The significant gains in quality of life and cognition achieved suggest that this practice can be valid to improve the conditions of the health of elderly in longterm institutions for the aged.
Key words: Health of Institutionalized Elderly. Communications Media Interpersonal Relations. Quality of life. Cognition. Depression.

\section{INTRODUÇÃO}

A educação permanente e a oportunidade de aprendizagens contínuas atendendo à necessidade de interação, participação e reconhecimento público podem permitir viver mais e melhor. ${ }^{1}$ Atividades desse âmbito têm sido desenvolvidas em grupos de terceira idade, universidades e centros de convivência. Instituições de longa permanência para idosos (ILPIs) abrigam uma população com condições de saúde distintas e uma parcela dos idosos não tem condições e/ ou possibilidades de frequentar esses espaços. $\mathrm{O}$ desenvolvimento de atividades com esse objetivo poderiam ser oferecidas nas ILPIs para possibilitar seus benefícios também a esses sujeitos.

$\mathrm{Na}$ ILPI, o indivíduo perde parte de sua posição social no mundo externo, o que pode significar a perda do "eu". ${ }^{2}$ Os elos estabelecidos pela interação com outros ambientes sociais são interrompidos, levando ao "enfraquecimento identitário", ao limitar a participação nos contextos que geravam sentimentos de pertencimento social e de validação subjetiva diante dos outros sujeitos, distanciando o sujeito cada vez mais desses cenários, diante das constantes revoluções tecnológicas. ${ }^{3}$

Parece haver um comprometimento maior da qualidade de vida (QV) e dos estados cognitivo e emocional em idosos de ILPI quando comparados aos que vivem com a família. ${ }^{4}$ Embora as
ILPIs possam ter caráter de confinamento e levem ao menor estímulo social do sujeito, por vezes se tornam a única alternativa viável para preservar mecanismos de sobrevivência diante das dificuldades socioeconômicas, afetivas e familiares encontradas na vida dos idosos. ${ }^{5} \mathrm{~A}$ depressão e os déficits cognitivos estão entre os principais problemas de saúde mental dos idosos, sendo muito comum que ambas as condições apareçam juntas e desencadeiem piora da QV, queda da funcionalidade, aumento no uso de serviços de saúde, aumento da morbidade e da mortalidade. $^{6}$

A demência culmina no declínio funcional progressivo e perda gradual da autonomia e da independência, e sua incidência e prevalência aumentam exponencialmente com a idade. ${ }^{7}$ As perdas cognitivas dependem mais da possibilidade de convívio social e das oportunidades que o idoso teve no curso de sua vida do que simplesmente um processo puramente biológico e de degradações progressivas. $^{8}$ A alfabetização é importante porque pode tornar as pessoas menos dependentes de terceiros para ler, redigir documentos, para nível de localização, locomoção por meio de transportes, acesso aos meios de comunicação e cuidados com a própria saúde. ${ }^{9}$

A baixa escolaridade pode estar associada com um declínio cognitivo mais rápido na velhice. Um 
estudo de coorte investigou a associação entre idade, sexo e escolaridade e o declínio cognitivo de 1.461 idosos por dez anos de seguimento. Os pesquisadores concluíram que o baixo nível de anos de estudo, gênero, idade e educação se associaram com o declínio cognitivo. ${ }^{10}$ Uma pesquisa sobre as habilidades cognitivas em indivíduos com idades maiores de 80 anos observou que o maior número de atividades de lazer e mais anos de escolaridade foram fatores preditivos de menores decréscimos. ${ }^{11}$

Uma revisão de literatura avaliou programas de promoção da saúde que desenvolviam atividades preventivas e/ou educativas para idosos em bases de dados, sites e periódicos especializados no período de 1990 a 2002. Os autores consideraram a avaliação em promoção da saúde do idoso como pouco desenvolvida no Brasil. A maioria das experiências no Brasil foi desenvolvida em serviços públicos de saúde, vinculada à assistência regular. Dentre as demais, duas foram realizadas em instituições de ensino via extensão universitária, uma pela Sociedade Brasileira de Geriatria e Gerontologia e outra sem vínculo institucional. ${ }^{12}$

Esta pesquisa fez uso de uma tecnologia de informação e comunicação (TIC) historicamente envolvida com o idoso desta geração, o rádio. O rádio é utilizado há bastante tempo no Brasil e constitui uma das TICs mais usadas pela população, sendo encontrada em 23.107 domicílios das cinco regiões do Brasil. A Região Sul é a que apresenta maior percentual destes equipamentos no domicílio, 91\%. ${ }^{13}$ As TICs parecem provocar alterações nas formas de aprendizado, na adaptação e até na percepção do sujeito em relação ao mundo e a ele mesmo, sendo a mídia um importante agente socializador ou educador. ${ }^{14}$

Refletindo sobre o contexto apresentado, este estudo teve como objetivo identificar possíveis mudanças na qualidade de vida, cognição e depressão de idosos advindas de oficinas de educação gerontológica mediadas por uma rádio-poste oferecidas em instituições de longa permanência para idosos.

\section{METODOLOGIA}

Estudo observacional realizado na cidade de Passo Fundo-RS, entre agosto de 2010 e outubro de 2011. Trata-se de pesquisa com natureza de dados qualitativa e quantitativa, que contemplou variáveis descritivas e de associação.

Primeiramente, foi realizado estudo piloto em um grupo de terceira idade da Universidade de Passo Fundo com 11 sujeitos, com quatro encontros de duas horas e com base nos resultados encontrados foram feitas as alterações necessárias. ${ }^{15} \mathrm{Na}$ primeira semana, foram coletados os dados sociodemográficos (análise das pastas e complementação de dados via entrevista) e realizada a entrevista individual para o rastreamento das condições verbais, cognitivas e o interesse de participação na pesquisa, sendo aplicados os questionários de qualidade de vida (Whoqol-old, Whoqol-bref e Flanagan), cognição (MEEM) e de depressão (EDG-15). Estes últimos foram reaplicados na última semana.

Para a escolha das ILPIs participantes, foram selecionadas oito instituições da cidade de Passo Fundo-RS que possuíam um controle sistemático do histórico de seus internos, tais como a organização dos documentos e histórico de rotinas de saúde, de admissão e alta dos idosos em pastas organizadas. Destas, apenas quatro se dispuseram a ajudar na pesquisa, sendo 161 internos entrevistados nestas ILPIs. Dentre estes, foram identificados 50 idosos em condições de participar, 20 não aceitaram responder aos questionários e sete foram excluídos por não seguirem a frequência dos encontros. Assim, a amostra selecionada por conveniência foi de 23 sujeitos.

Os critérios de inclusão foram possuir capacidade verbal preservada para falar ao microfone e capacidade cognitiva suficiente para o entendimento dos questionamentos e a elaboração de respostas plausíveis aos conteúdos. Os critérios de exclusão foram faltar a um dos encontros, não aceitar assinar o Termo de Consentimento Livre e Esclarecido e apresentar incapacidade para se 
expressar oralmente. Deficiências físicas, visuais e déficits parciais auditivos que ainda permitissem ouvir as falas com clareza e dialogar não eram considerados excludentes para a participação. Todos os sujeitos foram abordados de forma particular e individual.

Foram aplicados três questionários de QV, um para a suspeição de depressão, um para a avaliação do estado cognitivo, e um questionário semiestruturado para o conhecimento das características sociodemográficas, atividades de lazer e as TICs acessadas pelos participantes. $\mathrm{O}$ questionário semiestruturado foi desenvolvido por Pasqualotti ${ }^{16}$ e apresenta questões abertas e fechadas, relacionadas a quais TICs compunham o cenário do idoso, as atividades praticadas no tempo livre e seu significado para o entrevistado.

A escala de QV Whoqol-old foi desenvolvida pela Organização Mundial da Saúde e está estruturada em 24 itens atribuídos a seis facetas: "funcionamento do sensório", "autonomia", "atividades passadas, presentes e futuras", "participação social", "morte e morrer" e "intimidade". Cada uma destas facetas possui quatro itens; e para cada item, o pesquisado pode atribuir uma nota de 1 a 5. Optou-se por trabalhar com o escore bruto da faceta, que seria a soma dos pontos de cada uma somando uma pontuação de quatro a 20 pontos para cada faceta e variando de 24 a 120 pontos para o escore geral. ${ }^{17}$

A escala de QV Whoqol-bref foi elaborada a partir da Whoqol-100 e validada para o português por Fleck e colaboradores. ${ }^{18}$ São 26 questões, duas gerais sobre QV, e 24 outras que representam as 24 facetas ou domínios do instrumento Whoqol-100. Composta por quatro domínios: "físico", "psicológico", "relações sociais" e "meio ambiente", ${ }^{18,19}$ para cada questão nesta escala é conferida uma nota de 1 a 5 , sendo que nesta pesquisa os valores são dados em escores de 4 a 20 pontos.

A escala de QV de Flanagan foi criada pelo fisiologista americano John Flanagan, em 1970, contendo 15 itens que medem cinco domínios da QV: "bem-estar material e físico", "relacionamentos com as outras pessoas", "atividades social, comunitária e cívica", "desenvolvimento pessoal e realização" e "recreação". ${ }^{20}$ Possibilita conferir até sete pontos a cada questão, com escore total variando de 15 a 105 pontos. No Brasil, sua versão traduzida tem sido usada apresentando fidedignidade. ${ }^{21}$ Nesta pesquisa se adotou a média da pontuação de cada questão de determinada faceta, somando assim de 1 a 7 pontos de escore cada.

A Escala de Depressão Geriátrica (EDG), elaborada por Yesavage e colaboradores ${ }^{22}$ e adaptada para a população brasileira por Almeida \& Almeida, ${ }^{23,24}$ consiste de 15 indagações a respeito do que o idoso tem sentido na última semana, incluindo no dia da entrevista. As alternativas identificam situações de desamparo, inutilidade, desinteresse, aborrecimento, felicidade, entre outros. Quando o somatório for maior ou igual a cinco, há suspeição de depressão; já aquele que somou um escore de até quatro pontos terá diagnóstico negativo.

O Miniexame do Estado Mental (MEEM) é um instrumento adaptado por Folstein, Folstein \& $\mathrm{McHugh}^{25}$ que relaciona a escolaridade, a faixa etária e o escore obtido no instrumento para indicar presença ou não de déficit cognitivo por meio da soma da pontuação obtida em cada questão, do seguinte modo: (a) menos de quatro anos de ensino escolar: entre 60 e 69 anos e escore de até 22 pontos; entre 70 e 79 anos e escore de até 20 pontos; mais de 79 anos e escore de até 18 pontos; (b) ensino fundamental e escore de até 22 pontos; (c) nível médio ou superior e escore de até 23 pontos são consideradas situações de presença de déficit valores inferiores a estes limites. O MEEM permite analisar a cognição em cinco domínios - "orientação", "memória imediata", "atenção e cálculo", "evocação" e "linguagem" - ou usando apenas o somatório geral da pontuação. Foram analisados todos os domínios nesta pesquisa.

Não foi objetivo diagnosticar casos de demência e depressão, pois o uso isolado de tais instrumentos não seria capaz de responder a tal problemática, contudo, buscou-se 
rastrear sinais de depressão e de deficiência cognitiva. Para analisar os efeitos pré e pósprocesso de intervenção, foram realizadas estatísticas descritiva (média e desvio-padrão) e analítica, fazendo os testes não paramétricos de Mann-Whitney para a diferença de médias; Kolmogorov-Smirnov, para testar se as funções de distribuição das variáveis escolaridade, idade e tempo de institucionalização eram normais; Exato de Fisher, para comparar as frequências dos casos de depressão e de déficit cognitivo; Alfa de Cronbach, para verificar a consistência interna das escalas de QV utilizadas; Correlação linear de Spearman, para medir o grau de associação entre as variáveis, neste caso entre os domínios das escalas de QV, todos com nível de significância de $\mathrm{p} \leq 0,05$.

Foram elaborados roteiros de rádio pelos próprios pesquisadores com base no trabalho de um locutor de uma emissora de rádio AM da cidade de Passo Fundo-RS, para conferir uniformidade de intervenção entre as ILPIs abordadas. A intervenção incluiu oito encontros em cada instituição, duas vezes semanais, com duas horas cada, em dias e horários que se adaptavam à rotina interna da ILPI. Por meio dos debates que ocorriam no "momento da voz", procurou-se construir um conhecimento, ao mesmo tempo valorizando o teor da fala individual dos idosos e estimulando a tomada de posições. Do primeiro ao último dia, os debates se iniciavam com uma reflexão sobre o que cada um pensava, passando para a comunicação interpessoal, a comunicação em diferentes contextos e então se chegava aos meios de comunicação e às memórias ligadas ao rádio. Cada encontro se iniciava com a retomada do anterior e apresentação dos objetivos para o dia, buscando-se relacionar cada tema já abordado com o proposto para o dia.

A rádio foi instalada no fundo de uma sala, e os idosos se posicionaram em círculo, uns de frente para os outros. Caixas de som foram posicionadas nas salas e corredores das ILPIs para que todos pudessem ouvir a programação, que era estruturada da seguinte forma: 1) apresentação: cada participante se apresentava e mandava recado para os colegas, deixando sua mensagem; 2) previsão do tempo; 3) santos do dia; 4) frase do dia; 5) momento da voz: espaço de diálogo mediado por questões abertas e fechadas organizadas em torno dos temas; 6 ) horóscopo; 7) notícias; 8) radionovela; 9) mensagem do dia. Músicas solicitadas pelos participantes tocavam nos intervalos e os debates duravam em torno de 40 minutos, no mínimo. Quando possível, os participantes davam recados e mensagens durante a programação, de acordo com seu desejo, respeitando aqueles que não queriam se expor do mesmo modo.

Oprojeto obteve aprovação do Comitê de Ética em Pesquisa da Universidade de Passo Fundo, sob parecer $n^{\circ} 449 / 2010$, e todos os participantes assinaram o Termo de Consentimento Livre e Esclarecido.

\section{RESULTADOS}

Participaram 23 idosos, sendo 69,6\% mulheres; 56,5\% viúvos; com idade média de 78,1 anos (desvio-padrão de 9,4 anos); 5,8 anos de estudo (desvio-padrão de 4,7 anos), 91,3\% aposentados e com tempo de institucionalização médio de 36,6 meses (desvio-padrão de 39,5 meses). Somente o tempo de institucionalização não teve uma distribuição normal no teste de normalidade de Kolmogorov-Smirnov [idade $(\mathrm{p}=0,200)$, escolaridade $(\mathrm{p}=0,067)$ e tempo de institucionalização $(\mathrm{p} \leq 0,001)$, quando $\mathrm{p} \leq 0,05$.

Em relação ao cenário tecnológico das ILPIs, os idosos estavam habituados a acessar a televisão $(91,3 \%)$, o telefone $(82,6 \%)$, o rádio $(78,3 \%)$, o jornal impresso $(43,5 \%)$ e revistas ou livros $(43,5 \%)$. No lazer, costumavam receber visitas (95,7\%); andar pelo pátio (87\%); ir a igrejas ou participar de celebrações religiosas realizadas dentro da ILPI $(69,6 \%)$; costurar, bordar, tricotar ou pintar (34,8\%); jogar carta, dama ou dominó $(34,8 \%)$ e praticar jardinagem $(4,3 \%)$.

A memória imediata aumentou significativamente após a intervenção. Outras habilidades analisadas no MEEM e o escore total não apresentaram mudanças significativas estatisticamente. A pontuação obtida na EDG 
foi maior ao final da pesquisa, mas tanto os casos afirmativos para suspeição de depressão quanto para a presença de déficits cognitivos decresceram, ainda que não de forma significativa. Dos seis casos afirmativos de suspeição de depressão préintervenção, três permaneceram nessa condição após a intervenção - diferença estatística com nível de significância de $\mathrm{p}=0,956$. Ao mesmo tempo, ocorreu redução nos casos de déficits cognitivos de 14 para $11, \mathrm{p}=0,554$, no teste exato de Fisher. A tabela 1 mostra os escores totais da EDG e do MEEM.

Tabela 1. Pontuação da Escala de Depressão Geriátrica e Miniexame do Estado Mental dos processos de pré e pós-intervenção. Passo Fundo-RS, 2010-2011.

\begin{tabular}{|c|c|c|c|c|c|}
\hline & Variáveis & Intervenção & Média & Desvio-padrão & $\mathrm{p}$ \\
\hline \multirow{2}{*}{ Ф્ } & \multirow{2}{*}{ Pontuação EDG } & Pré-intervenção & 2,7 & 2,1 & \multirow{2}{*}{0,459} \\
\hline & & Pós-intervenção & 2,8 & 2,5 & \\
\hline \multirow{12}{*}{ 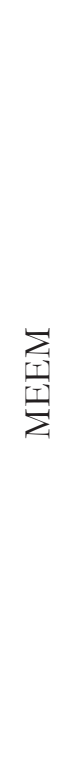 } & \multirow{2}{*}{ Orientação } & Pré-intervenção & 6,6 & 2,1 & \multirow{2}{*}{0,657} \\
\hline & & Pós-intervenção & 6,9 & 2,4 & \\
\hline & \multirow{2}{*}{ Memória imediata } & Pré-intervenção & 2,2 & 1,0 & \multirow{2}{*}{$0,037^{*}$} \\
\hline & & Pós-intervenção & 2,7 & 0,8 & \\
\hline & \multirow{2}{*}{ Atenção e cálculo } & Pré-intervenção & 1,6 & 1,8 & \multirow{2}{*}{0,537} \\
\hline & & Pós-intervenção & 2,0 & 1,9 & \\
\hline & \multirow{2}{*}{ Evocação } & Pré-intervenção & 1,3 & 1,1 & \multirow{2}{*}{0,784} \\
\hline & & Pós-intervenção & 1,4 & 1,4 & \\
\hline & \multirow{2}{*}{ Linguagem } & Pré-intervenção & 6,8 & 1,3 & \multirow{2}{*}{0,443} \\
\hline & & Pós-intervenção & 7,0 & 1,3 & \\
\hline & \multirow{2}{*}{ Pontuação MEEM } & Pré-intervenção & 18,5 & 5,2 & \multirow{2}{*}{0,327} \\
\hline & & Pós-intervenção & 20,0 & 5,9 & \\
\hline
\end{tabular}

*Nível de significância de $\mathrm{p} \leq 0,05$. Teste de Mann-Whitney.

A análise da QV por meio da escala Whoqolbref revelou um incremento em todos os seus domínios, sem significância estatística, porém considerável no que se refere à avaliação geral da saúde, questão que investiga o quanto a pessoa está satisfeita com sua saúde (tabela 2). 
Tabela 2. Resultados dos domínios da Escala de Qualidade de Vida Whoqol-bref obtidos nos processos de pré e pós-intervenção. Passo Fundo-RS, 2010-2011.

\begin{tabular}{lcccc}
\hline \multicolumn{1}{c}{ Variáveis } & Intervenção & Média & Desvio-padrão & $\mathrm{p}^{*}$ \\
\hline \multirow{2}{*}{ Físico } & Pré-intervenção & 13,6 & 1,8 & 0,239 \\
& Pós-intervenção & 14,1 & 1,4 & \\
\multirow{2}{*}{ Psicológico } & Pré-intervenção & 14,3 & 2,1 & 0,808 \\
& Pós-intervenção & 14,4 & 2,5 & \\
Relações sociais & Pré-intervenção & 16,2 & 2,3 & 0,358 \\
\multirow{2}{*}{ Meio ambiente } & Pós-intervenção & 16,9 & 2,8 & \\
& Pré-intervenção & 16,1 & 2,5 & 0,912 \\
Escore geral & Pós-intervenção & 16,3 & 2,2 & \\
& Pré-intervenção & 14,8 & 2,5 & 0,132 \\
Avaliação geral & Pós-intervenção & 15,7 & 2,4 & \\
& Pré-intervenção & 16,6 & 4,5 & 0,558 \\
Avaliação geral da saúde & Pós-intervenção & 17,1 & 4,7 & 0,057 \\
& Pré-intervenção & 17,1 & 4,7 & \\
\hline
\end{tabular}

*Nível de significância de $\mathrm{p}<0,05$. Teste de Mann-Whitney.

Calculando o coeficiente de correlação de Spearman, nos resultados pré-intervenção não ocorreram correlações significativas entre a avaliação geral da saúde com os outros domínios. O aumento da percepção de saúde pareceu estar correlacionado com os domínios "físico" $(\mathrm{p}=0,021)$, "psicológico" $(\mathrm{p}=0,036)$ e "meio ambiente" ( $\mathrm{p}=0,028)$ após a intervenção.
$\mathrm{Na}$ escala de QV Whoqol-old, o domínio "intimidade" aumentou de forma significativa depois da experiência com as oficinas. Este domínio avalia a capacidade de se ter relações pessoais e íntimas, o quanto a pessoa sente que tem oportunidades para amar e ser amada, e o quanto sente que tem companheirismo e amor em sua vida (tabela 3). 
Tabela 3. Resultados dos processos de pré e pós-intervenção obtidos na Escala de Qualidade de Vida Whoqol-old. Passo Fundo-RS, 2010-2011.

\begin{tabular}{lcccc}
\hline \multicolumn{1}{c}{ Variáveis } & Intervenção & Média $^{*}$ & Desvio-padrão & p $^{* *}$ \\
\hline Funcionamento & Pré-intervenção & 17,3 & 3,5 & 0,537 \\
do sensório & Pós-intervenção & 17,5 & 2,7 & \\
\multirow{2}{*}{ Autonomia } & Pré-intervenção & 14,0 & 4,4 & 0,163 \\
Atividades passadas, & Pós-intervenção & 13,9 & 4,7 & \\
presentes e futuras & Pré-intervenção & 15,7 & 3,8 & 0,774 \\
\multirow{2}{*}{ Participação social } & Pós-intervenção & 15,2 & 4,3 & \\
& Pré-intervenção & 14,7 & 4,3 & 0,113 \\
Morte e morrer & Pós-intervenção & 15,8 & 4,3 & \\
& Pré-intervenção & 18,0 & 3,4 & 0,404 \\
Intimidade & Pós-intervenção & 17,5 & 4,4 & \\
& Pré-intervenção & 14,8 & 4,8 & $0,023 * *$ \\
Escore geral & Pós-intervenção & 15,8 & 3,4 & 0,226 \\
& Pré-intervenção & 94,6 & 2,6 & \\
\hline
\end{tabular}

*Média aritmética a partir do escore bruto das facetas.**Nível de significância de $\mathrm{p}<0,05$. Teste de Mann-Whitney.

A "intimidade" teve correlação significativa com os domínios "autonomia" ( $\mathrm{p}=0,011)$, "atividades passadas, presentes e futuras" $(p=0,003)$ e "participação social" ( $p=0,001)$, quando analisada a pré-intervenção. Após a intervenção, essa correlação significativa se manteve ["autonomia" ( $\mathrm{p}=0,001)$, "atividades passadas, presentes e futuras" ( $\mathrm{p}=0,007)$ e "participação social" $(\mathrm{p}=0,013)]$. Estes se referem à independência na velhice, respectivamente, e, portanto, descrevem até que ponto se é capaz de viver de forma autônoma e tomar suas próprias decisões, a satisfação sobre conquistas na vida e coisas a que se anseia, e delineiam a participação em atividades do cotidiano, especialmente na comunidade.

Na escala de QV de Flanagan, o domínio "recreação", que avalia a satisfação diante da participação em atividades de recreação, lazer e de socialização, foi significativamente maior após a intervenção (tabela 4). 
Tabela 4. Resultados obtidos nos processos pré e pós-intervenção na Escala de Qualidade de Vida de Flanagan. Passo Fundo-RS, 2010-2011.

\begin{tabular}{ccccc}
\hline Variáveis & Intervenção & Média & Desvio-padrão & p \\
\hline \multirow{2}{*}{ BMF } & Pré-intervenção & 5,5 & 1,1 & 0,452 \\
& Pós-intervenção & 5,8 & 0,7 & \\
\multirow{2}{*}{ ROP } & Pré-intervenção & 5,9 & 1,0 & 0,641 \\
& Pós-intervenção & 6,1 & 0,7 & \\
\multirow{2}{*}{ ASCC } & Pré-intervenção & 5,6 & 1,2 & 0,514 \\
& Pós-intervenção & 5,9 & 0,7 & \\
\multirow{2}{*}{ DRP } & Pré-intervenção & 6,0 & 0,7 & 0,956 \\
& Pós-intervenção & 6,0 & 0,8 & \\
& Pré-intervenção & 5,6 & 0,7 & $0,044^{*}$ \\
\hline
\end{tabular}

*Nível de significância de $\mathrm{p}<0,05$. Teste de Mann-Whitney. BMF= bem-estar material e físico; $\mathrm{ROP}=$ relacionamentos com as outras pessoas; ASCC = atividades social, comunitária e cívica; $\mathrm{DRP}=$ desenvolvimento e realização pessoal; $\mathrm{RE}=$ recreação.

Da investigação da correlação entre a "recreação" e os outros domínios desta escala, no período pré-intervenção havia correlação significativa com o "bem-estar material e físico" $(\mathrm{p}=0,001)$, "relacionamentos com outras pessoas" $(\mathrm{p}=0,033)$, "atividades social, comunitária e cívica" $(\mathrm{p}<0,001) \mathrm{e}$ "desenvolvimento e realização pessoal" $(p<0,001)$. Nos resultados pós-intervenção, a "recreação" correlacionou-se com a qualidade dos "relacionamentos com as outras pessoas" $(\mathrm{p}=0,003)$ e com o "desenvolvimento e realização pessoal” $(\mathrm{p}<0,001)$, que, respectivamente, estudam a satisfação com os relacionamentos com os pais, amigos, parentes, com a satisfação do ter e criar filhos, com relacionamentos importantes como com namorado, esposo ou pessoa relevante e o autoconhecimento de potenciais e limitações, a satisfação com a capacidade de aprender, de trabalhar e de se expressar.

Os instrumentos de QV utilizados nesta pesquisa apresentaram valores indicativos de confiabilidade para a amostra investigada, por meio do coeficiente alfa de Cronbach, e os valores de $\mathrm{p}$ encontrados pré e pós-intervenção foram, respectivamente, na Whoqol-old, 0,816 e 0,768; na Whoqol-bref, 0,856 e 0,862; e na Flanagan, 0,885 e 0,847 .

\section{DISCUSSÃO}

Grande parte dos domínios avaliados pelas escalas de QV e de investigação de déficits cognitivos e de depressão aumentou após a intervenção, mesmo que não tenham alcançado diferença estatística significativa. Contudo, três domínios da escala de QV Whoqol-old decresceram sua pontuação: "autonomia", "atividades passadas, presentes e futuras" e "morte e morrer". Uma hipótese seria que os participantes poderiam ter se tornado mais críticos com as reflexões desenvolvidas nas oficinas.

Dessa forma, passando a perceber limitações na liberdade de escolhas nas atividades diárias da vida, demonstrando insegurança em relação à morte que se aproxima, insatisfação com $\mathrm{O}$ número de oportunidades para novas realizações, quanto ao reconhecimento recebido pelas ações desenvolvidas antes da institucionalização, com o que alcançou na vida, ou ainda, não esperando um futuro melhor que o atual. Além desta hipótese, podem ter ocorrido acontecimentos particulares durante a pesquisa, que poderiam ter corroborado para esta redução. Ainda pode-se estipular que a atividade proposta não conseguiu atingir todos os domínios da QV, mas que 
conseguiu aumentar os escores gerais das escalas ao incrementar principalmente a "autopercepção da saúde", a "intimidade" e a "recreação", podendo contrabalançar os domínios que não foram abordados diretamente, como o "funcionamento do sensório" e o "físico".

Os idosos das ILPIs demonstraram aumento significativo estatisticamente nos domínios "intimidade" na escala de QV Whoqol-old, "recreação" na escala de QV Flanagan e na "memória imediata" no MEEM. No geral, nas escalas de QV Whoqol-bref e Whoqol-old foram obtidos escores menores que outras amostras compostas tanto de idosos ambulatoriais quanto de ILPI, ${ }^{25,26,27}$ ao passo que, na escala de QV de Flanagan, a pontuação foi maior quando comparada com amostras significativas existentes na literatura. ${ }^{28}$ Esses achados podem refletir a heterogeneidade dessa faixa etária, pois a idade não parece ser o determinante principal das condições de cognição, depressão e QV.

A exemplo disso, Maués e colaboradores, ${ }^{26}$ ao compararem a QV entre 69 idosos separados em dois grupos, os jovens (idade entre $60 \mathrm{e}$ 70 anos) e muito idosos (85 anos ou mais) não encontraram diferença estatística significativa, pela escala de QV Whoqol-old. No grupo dos idosos jovens, a média do escore geral foi de 84,1 , e nos muito idosos, 83,3. Com base neste achado, os autores concluíram que a QV não parece decair com a idade. Os participantes deste estudo apresentaram menores escores de QV na avaliação inicial sem intervenção do que os idosos ambulatoriais. Ao transformar os valores apresentados de zero a 100 (escores transformados das facetas), seriam obtidas como médias dos escores gerais nas quatro ILPIs investigadas: na ILPI A, 68,8; na ILPI B, 71,3; na ILPI C, 72,0; e na ILPI D, 81,9 após a intervenção passando para, respectivamente, 78,1; 82,9; 76,8; e 73,8.

Esses escores parecem confirmar os achados da literatura que creem numa diminuição da QV com a institucionalização em ILPI, ${ }^{4,27}$ mas nesse cenário outros fatores podem contribuir para a redução ou a manutenção de boas condições de
QV nesse ambiente. Tavares e colaboradores ${ }^{28}$ investigaram a QV em 1.303 idosos com hipertensão arterial de uma comunidade usando as escalas de QV Whoqol-old e Whoqol-bref. Os resultados primeiramente mostraram que a escolaridade dos entrevistados na maioria ficava entre um e cinco anos de estudo (59,2\%), o que fica muito próximo do encontrado nesta pesquisa, em que a média da escolaridade foi de 5,8 anos, com desvio-padrão de 4,7 anos de estudo.

A baixa escolaridade pode estar associada com um declínio cognitivo mais rápido na velhice do que o encontrado em amostras de idosos com mais anos de estudo. Castro-Costa e colaboradores, ${ }^{11}$ num estudo de coorte, investigaram a associação entre idade, sexo e escolaridade e o declínio cognitivo de 1.461 idosos por dez anos de seguimento. Os pesquisadores concluíram que o baixo nível de anos de estudo, gênero, idade e educação se associou com o declínio cognitivo. Para estes, as mulheres e os indivíduos com maior escolaridade apresentaram resultados mais altos no MEEM na linha de base, e os idosos mais velhos (75 anos ou mais) apresentaram pontuações mais baixas.

Em relação aos achados obtidos no MEEM e na EDG quando comparados à literatura, ${ }^{4}$ a amostra deste estudo apresentou estado cognitivo no geral baixo e poucos casos de depressão entre os investigados, e embora não haja mudanças significativas estatisticamente, podem ser resultados positivos. Dos 23 participantes, seis tinham suspeição de depressão, caindo para três ao final da pesquisa, com pontuação média e desviopadrão inicial de $2,7(\mathrm{dp}=2,1)$, passando para 2,8 $(\mathrm{dp}=2,5)$ dos 15 pontos possíveis, ao passo que a pontuação obtida no MEEM passou de 18,5 para 20,0 pontos, com ganho estatisticamente significativo para o domínio "memória imediata", que consiste na memorização de três palavras apresentadas no momento da entrevista. A amostra não representou fielmente a condição cognitiva da população investigada nas quatro ILPIs. Apenas 14,3\% dos 161 idosos que residiam nas quatro ILPIs investigadas responderam aos 
questionários, pois a maior parte não apresentava condições de respondê-los. Esse achado figura uma realidade comum em ILPI, já que as demências estão entre as principais causas de institucionalização. ${ }^{4}$

As atividades oferecidas no interior da ILPI também podem corroborar a QV dos idosos. Nesta pesquisa, os idosos na maior parte recebiam visitas de parentes, tinham contato com diversas e necessárias especialidades médicas, serviço psicológico, odontológico, nutricional, de enfermagem e fisioterapêutico, entre outros. Porém, o ganho de QV encontrado pode ter alguma relação com os ganhos de recreação, entretenimento e diversão. As atividades oferecidas pelos pesquisadores, desde as notícias, as músicas e os debates, foram escritos visando assuntos do interesse dos participantes, permitindo que interviessem na programação, contassem histórias de vida e experiências, o que lhes agradava e desagradava na ILPI. A partir dessa atitude, percebeu-se não se tratar simplesmente de oferecer atividades, mas de considerar os interesses dos idosos e a importância da ludicidade para gerar ganhos nos níveis cognitivos, emocionais e na QV. ${ }^{29}$

Plati e colaboradores ${ }^{29}$ avaliaram a frequência de sintomas depressivos e o desempenho cognitivo de idosos não institucionalizados; institucionalizados que recebiam terapia ocupacional, jogos, artes e desenhos, ginástica e visitas regulares de parentes, einstitucionalizados que não tinham as mesmas atividades. Foram 120 idosos, dos quais 83 institucionalizados, em que $54,2 \%$ dos indivíduos apresentavam sintomas depressivos, valor menor que a proporção não institucionalizada, em que estes sintomas apareceram em 18,9\% dos sujeitos. As médias dos escores obtidos na EDG foram de 4,2 $(\mathrm{dp}=3,4)$ nos institucionalizados com atividades; 5,5 $(\mathrm{dp}=3,6)$ nos institucionalizados sem atividades; e 2,4 $(\mathrm{dp}=2,7)$ nos não institucionalizados. No MEEM, os autores encontraram escores de 24,2 $(\mathrm{dp}=3,2)$ nos idosos não institucionalizados; $24,2 \quad(\mathrm{dp}=4,1)$ nos institucionalizados com atividades; e 21,7 $(\mathrm{dp}=4,1)$ nos institucionalizados sem atividades.

Santos e colaboradores ${ }^{30}$ utilizaram a escala de QV de Flanagan para avaliar a QV de 128 idosos de uma comunidade, e a média dos escores obtidos nessa amostra foi de 74,3, com coeficiente de confiabilidade alpha igual a 0,764 . Os autores ${ }^{30}$ afirmaram a eficiência do instrumento, pois, segundo eles, um valor de alpha maior que 0,600 significaria a concordância entre o instrumento e a população-alvo. Em comparação, foram encontrados neste estudo média geral e desviopadrão de 87,3 (dp=10,9) e coeficiente de confiabilidade alpha de Cronbach igual a 0,885 pré-intervenção e 0,847 na avaliação final.

Oliveira, Gomes \& Paiva ${ }^{27}$ compararam a QV de 70 idosos funcionalmente independentes e residentes em instituições e 210 não institucionalizados, utilizando a escala de QV Whoqol-bref, e verificaram uma pior QV no grupo dos institucionalizados quando comparados aos não institucionalizados, que apresentaram melhores pontuações em todos os domínios do instrumento. Os autores observaram uma associação entre institucionalização e todos os domínios da QV e concluíram que a institucionalização de idosos pode ser fator determinante na perda da QV.

Acredita-se que a institucionalização pode estar fortemente envolvida na diminuição da QV, porém a pesquisadora Ana Amélia Camarano, em entrevista à revista IstoÉ, afirma que em muitos casos as ILPIs podem melhorar a QV dos idosos, principalmente de acordo com a expectativa que este tinha antes da institucionalização, obtendo ganhos, sobretudo por meio da socialização com pessoas na mesma situação, enquanto alguns podem tornar-se revoltosos quando internados contra a vontade. ${ }^{31}$

Definir a velhice apenas como a última fase da vida pode contribuir para a segregação da pessoa idosa, não só em ILPI, mas também no próprio seio da sociedade, pois, se assim o fosse, seria diminuída a motivação para viver 
essa etapa. ${ }^{32}$ Segundo Both, ${ }^{1}$ "a constituição do ser humano, independentemente da idade, reproduz em seu desenvolvimento as formas de linguagem, de entendimento, de habitar e a afetividade representada e expressa em seu ambiente social"'.

Uma correlação entre a recreação, o desenvolvimento e realização pessoal e a interação com outras pessoas pode ser identificada entre os domínios da escala de QV de Flanagan. Foi encontrada uma correlação significativa entre os domínios "desenvolvimento e realização pessoal" e "recreação" $(p<0,001)$, e ainda, entre os domínios "desenvolvimento e realização pessoal" e "relação com as outras pessoas" ( $\mathrm{p}=0,023)$. Both ${ }^{1}$ sugere uma possível contribuição das atividades de lazer e das relações interpessoais proporcionadas dentro das instituições para o desenvolvimento humano e a autorrealização dos sujeitos.

Na escala de QV de Flanagan, o domínio "relacionamento com outras pessoas" envolve a percepção de qualidade do diálogo, da presença e da ajuda recebida de parentes, família, amigos e pessoas relevantes na vida do sujeito. Já o domínio "recreação" abrange a qualidade das atividades de recreação, de lazer, das oportunidades e da capacidade de socialização. Já o domínio "desenvolvimento e realização pessoal" envolve a autopercepção da capacidade de aprender, o autoconhecimento dos potenciais e limitações e a capacidade para o trabalho e ao diálogo.

Em virtude das condições de saúde apresentadas pelos idosos das ILPIs selecionadas houve dificuldade em encontrar amostras significativas estatisticamente, constituindo uma limitação do estudo. Recomenda-se que esta pesquisa seja realizada em ILPIs com amostras com maior poder de generalização dos dados.

\section{CONCLUSÕES}

Ocorreram incrementos significativos estatisticamente na qualidade de vida, cognição e depressão, nos domínios "recreação" da escala de qualidade de vida de Flanagan, "intimidade" do Whoqol-old e "memória imediata" do Miniexame do Estado Mental. Embora o número de casos de déficits cognitivos e de depressão tenha diminuído entre os participantes, não se obteve significância estatística.

Os resultados desta pesquisa sugerem ser necessário estimular cognitivamente os idosos nas ILPIs para poder beneficiá-los com ganhos não apenas cognitivos, mas emocionais e de qualidade de vida. Este estudo pode contribuir para corroborar a importância da educação permanente voltada para as necessidades de cada idoso de maneira adaptada aos diversos cenários sociais e culturais.

\section{REFERÊNCIAS}

1. Both A. Educação gerontológica: posições e proposições. Erechim: São Cristóvão, 2001.

2. Watanabe HAW. Instituições de Longa Permanência para idosos (ILPI). In: São Paulo (Estado), Fundação Padre Anchieta. Rede de atenção à pessoa idosa. São Paulo: SEADS; Fundação Pe. Anchieta; 2009. p. 11-31.

3. Pasqualotti A, Barone AC, Doll J, Camozzato TM. Significação dos processos de comunicação e interação de pessoas idosas residentes numa instituição de longa permanência. Rev Bras Ciênc Envelhec Hum 2009;6(1):20-33.

4. Carneiro RS, Falcone E, Clark C, Del Prette Z, Del Prette A. Qualidade de vida, apoio social e depressão em idosos: relação com habilidades sociais. Psicol Reflex Crític 2007;20(2):229-37.

5. Araújo LF, Coutinho MPL, Saldanha AAW. Análise comparativa das representações sociais da velhice entre idosos de instituições geriátricas e grupos de convivência. Psico 2005;36(2):197-204.

6. Ávila R, Bottino CMC. Atualização sobre alterações cognitivas em idosos com síndrome depressiva. Rev Bras Psiquiatr 2006;28(4):316-20. 
7. BRASIL. Ministério da Saúde, Secretaria de Atenção à Saúde, Departamento de Atenção Básica. Envelhecimento e saúde da pessoa idosa [Internet]. Brasília: Ministério da Saúde; 2006 [acesso em 3 dez 2013]. 191 p. (Série A. Normas e Manuais Técnicos) (Caderno de Atenção Básica, n. 19) Disponível em: http://bvsms.saude.gov.br/bvs/publicacoes/ abcad19.pdf

8. Gamburgo LJL, Monteiro MIB. Envelhecimento e linguagem: algumas reflexões sobre aspectos cognitivos na velhice. Rev Kairós 2007;10(1):35-49.

9. Instituto Brasileiro de Geografia e Estatística. Síntese de indicadores sociais: uma análise das condições de vida da população brasileira 2008. Estudos \& Pesquisas: informação demográfica e socioeconômica n. 23 [Internet]. 2008 [Acesso em 2 fev 2010]. 280 p. Disponível em: http://www.ibge. gov.br/home/estatistica/populacao/condicaodevida/ indicadoresminimos/sinteseindicsociais2008/indic_ sociais2008.pdf

10. Castro-Costa E, Dewey ME, Uchôa E, Firmo JO, Lima-costa MF, Stewart R. Trajectories of cognitive decline over 10 years in a Brazilian elderly population: the Bambuí Cohort Study of Aging. Cad Saúde Pública 2011;27(supl 3):345-50.

11. Argimon IIL, Stein LM. Habilidades cognitivas em indivíduos muito idosos: um estudo longitudinal. Cad Saúde Pública 2005;21(1):64-72.

12. Assis M, Hartz ZMA, Valla VV. Programas de promoção da saúde do idoso: uma revisão da literatura científica no período de 1990 a 2002. Ciênc Saúde Coletiva 2004;9(3):557-81.

13. CETIC.br. TIC domicílios e usuários 2010 - total Brasil [Internet]. São Paulo: Centro de Estudos sobre as Tecnologias de Informação e Comunicação; 2005 - [acesso em 2 jan 2012]. Disponível em: http://cetic. br/usuarios/tic/2010-total-brasil/rel-geral-00.htm

14. Setton MGJ. A particularidade do processo de socialização contemporâneo. Tempo Soc 2005;17(2):335-50.

15. Rocha JP, Galvan TC, Silveira MM, Kumper DA, Klein OJ, Pasqualotti A. Educação gerontológica e qualidade de vida: uma proposta de prática interdisciplinar de promoção à saúde por meio da interação dialógica. Rev Contexto \& Saúde 2011;10(20):245-52.

16. Pasqualotti A. Comunicação, tecnologia e envelhecimento: significação da interação na era da informação [tese]. Porto Alegre: Universidade Federal do Rio Grande do Sul, Centro Interdisciplinar de Novas Tecnologias na Educação; 2008.
17. Power M, Schmidt S; Chachamovich E, tradutor; Fleck MPA. Organização Mundial da Saúde: manual WHOQOL-old [Internet]. 1998 [acesso em 22 jun 2010]. Disponível em: http://www.ufrgs.br/psiq/ WHOQOL-LD\%20Manual\%20POrtugues.pdf

18. Fleck MPA, Louzada S, Xavier M, Chachamovich E, Vieira G, Santos L, et al. Aplicação da versão em português do instrumento abreviado de avaliação da qualidade de vida “WHOQOL-Bref”. Rev Saúde Pública 2000;34(2):178-83.

19. World Health Organization. Whoqol-bref: introduction, administration, scoring and generic version of the assessment. Field Trial Version [Internet]. Geneva: WHO; 1996 [Acesso em 29 jan 2012]. 18 p. Disponível em: http://www.who.int/ mental_health/media/en/76.pdf

20. Burckhardt CS, Anderson KL. Review. The quality of life scale (QOLS): Reliability, validity and utilization. Health Qual Life Outcomes 2003;1(1):1-7.

21. Dantas RAS, Góis CFL, Silva LM. Utilização da versão adaptada da escala de qualidade de vida de Flanagan em pacientes cardíacos. Rev Latinam Enferm 2005;13(1):15-20.

22. Yesavage JA, Brink TL, Rose TL, Lum O, Huang $\mathrm{V}$, Adey M, et al. Development and validation of a geriatric depression screening scale: A preliminary report. J Psychiatr Res 1983;17(1):37-49.

23. Almeida OP, Almeida SA. Confiabilidade da versão brasileira da Escala de Depressão em Geriatria (GDS) versão reduzida. Arq Neuropsiquiatria 1999;57(2b):421-26.

24. Almeida OP, Almeida SA. Short versions of the geriatric depression scale: a study of their validity for the diagnosis of a major depressive episode according to ICD-10 and DSM-IV. Int J Geriatric Psychiatry 1999;14(10):858-65.

25. Folstein MF, Folstein SE, McHugh PR. Mini Mental State: A practical method for grading the cognitive state of patients for the clinical. J Psychiatr Res 1975;12(3):189-98.

26. Maués CR, Paschoal SMP, Jaluul O, França CC, Jacob Filho W. Avaliação da qualidade de vida: comparação entre idosos jovens e muito idosos. Rev Soc Bras Clín Méd 2010;8(5):405-10.

27. Tavares DMS, Martins NPF, Diniz MA, Dias FA, Santos NMF. Qualidade de vida de idosos com hipertensão arterial. Rev Enferm UERJ 2011;19(3):438-44.

28. Plati MCF, Covre P, Lukasova ECM. Depressive symptoms and cognitive performance of the elderly: relationship between institutionalization and activity programs. Rev Bras Psiquiatr 2006;28(2):118-21. 
29. Santos SR, Santos IBC, Fernandes MGM, Henriques MERM. Qualidade de vida do idoso na comunidade: aplicação da escala de Flanagan. Rev Latinam Enferm 2002;10(6):757-64.

30. Oliveira ERA, Gomes MJ, Paiva KM.

Institucionalização e qualidade de vida de idosos da região metropolitana de Vitória - ES. Esc Anna Nery Rev Enferm 2011;15(3):518-23.
31. Azevedo S. Como se estivessem em casa. ISTO É Independente 201017 mar;2105(34):54-9. (Seção Comportamento).

32. Vagetti GC, Weinheimer MS, Oliveira V. Atendimento integral à saúde do idoso residente em instituição de longa permanência: uma experiência. Estud Interdiscip Envelhec 2007;11:53-66.

Recebido: 26/10/2012

Revisado: $12 / 12 / 2013$

Aprovado:17/12/2013 